\title{
Muon Ionization Cooling Experiment (MICE): Results \& Prospects
}

\section{Paolo Franchini on behalf of the MICE collaboration ${ }^{a, *}$ \\ ${ }^{a}$ Department of Physics, Blackett Laboratory, Imperial College London, Exhibition Road, London, UK}

E-mail: p.franchini@imperial.ac.uk

\begin{abstract}
A high-energy muon collider could be the most powerful and cost-effective collider approach in the multi-TeV regime, and a neutrino source based on decay of an intense muon beam would be ideal for measurement of neutrino oscillation parameters. Muon beams may be created through the decay of pions produced in the interaction of a proton beam with a target. The muons are subsequently accelerated and injected into a storage ring where they decay producing a beam of neutrinos, or collide with counter-rotating antimuons. Cooling of the muon beam would enable more muons to be accelerated resulting in a more intense neutrino source and higher collider luminosity. Ionization cooling is the novel technique by which it is proposed to cool the beam. The Muon Ionization Cooling Experiment collaboration has constructed a section of an ionization cooling cell and used it to provide the first demonstration of ionization cooling. Here the observation of ionization cooling is described. The cooling performance is studied for a variety of beam and magnetic field configurations. The future outlook for muon ionization cooling demonstrations is discussed.
\end{abstract}

\footnotetext{
*** The European Physical Society Conference on High Energy Physics (EPS-HEP2021), *** *** 26-30 July $2021 * * *$

*** Online conference, jointly organized by Universität Hamburg and the research center DESY ***
}

\footnotetext{
${ }^{*}$ Speaker
} 


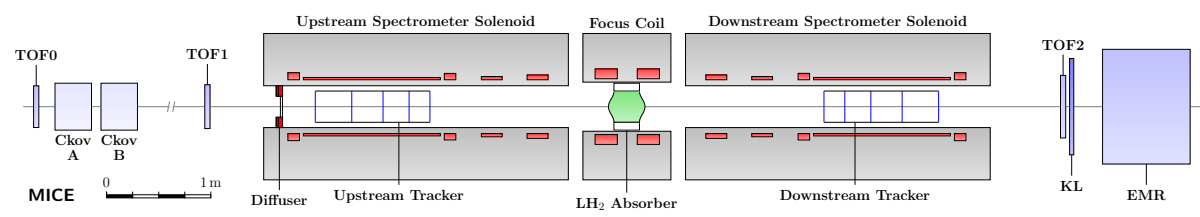

Figure 1: Schematic of MICE, including the PID detectors, the tracking apparatus immersed in the magnetic field within the cooling channel. Muons travel from left to right.

\section{Muon cooling}

Existing and proposed collider facilities have a huge footprint, while a muon collider has potential to explore physics at the highest energy in a relatively smaller scale. Muons are fundamental particles so the entire energy is available for production of secondary particles, rather than being spread among constituent quarks, as happens for protons. The synchrotron radiation is suppressed by the high muon mass, so muons can be accelerated to high energy in ring accelerators, unlike in electron-positron colliders. Muons are produced as tertiary particles: pions are produced by firing protons onto a target; pions decay to muons, therefore the resultant beam has high emittance. A suitable luminosity can be reached only reducing the muon emittance on a time scale compatible with the muon lifetime. Ionization cooling is the only identified technique that can be used to cool muons to provide a beam suitable for a muon collider. In muon ionization cooling, muons are passed through energy-absorbing material where the transverse and longitudinal momentum is reduced, decreasing the normalised beam emittance and "cooling" the beam.

\section{MICE}

The Muon Ionization Cooling Experiment (MICE) was designed to demonstrate the ionization cooling principle. A schematic of MICE is shown in fig. 1. Pions were produced from the halo of ISIS synchrotron proton beam hitting a target; next were guided into the cooling apparatus by sets of quadrupoles, dipoles and a solenoid. Momentum of the resultant beam was selected by the dipoles. A variable thickness diffuser at the entry of the cooling channel selected the incident beam emittance. The beam was passed into a solenoid focussing channel. Spectrometer Solenoid modules were located upstream and downstream of a Focus Coil module within which the absorber was placed providing a tight focus in both transverse planes suitable for ionisation cooling. Liquid hydrogen and lithium hydride absorbers were used. A pair of Time-of-Flight (TOF) detectors was used to estimate the particle velocity. Scintillating fibre trackers upstream and downstream of the experiment in fields of up to $4 \mathrm{~T}$ enabled characterisation of particles' position and momentum before and after passing through the cooling section. By comparing velocity and momentum, pion and electron were rejected. Muons were passed through the experiment one-by-one and an ensemble of muons was accumulated over hours. A number of configurations were studied having different incident momenta and emittances. Absorbers were changed every few weeks. The apparatus proved to be stable over this time [1]. The experiment was modelled using a bespoke Geant4-based simulation [2]. 


\section{Results}

Transverse amplitude distributions were found by counting the number of muons sitting within hyper-ellipsoids of varying sizes in four dimensional phase space upstream and downstream. Enhancement in the number of particles at low amplitude when passing through the absorber was indicative of an increase in the number of muons in the beam core (cooling). A decrease in the number of particles at high amplitude was indicative of either migration towards the beam core or scraping.

The amplitude distributions are shown in fig. 2 [3], for beams having nominal momentum of $140 \mathrm{MeV} / c$ and incident RMS emittances of 4, 6 and $10 \mathrm{~mm}$ (4-140, 6-140 and 10-140 respectively). Beam was passed through the liquid hydrogen vessel both in an empty (Empty LH2) and full (Full LH2) configuration. The beam was also passed through the experiment with No absorber and the lithium hydride ( $\mathrm{LiH})$ absorber. In the Empty LH2 and No absorber configuration, the core of the beam was observed to have the same number of particles both upstream and downstream of the absorber. The tail of the distribution was observed to be depleted above about $30 \mathrm{~mm}$ amplitude due to beam scraping on the beam pipe. In the Full LH2 and LiH case, there was a significant enhancement in the number of muons downstream of the absorber in the beam core, interpreted as the signal for cooling: the beam core density has increased. The ratios of the amplitude distributions are shown in fig. 3 together with the simulated cooling performance. Where the number of muons has increased, the ratio is more than 1 . Where the number of muons has decreased, the ratio is less than 1. A clear signal for the enhancement in core density is observed for settings where an absorber was installed. The simulated cooling performance shows good agreement with the measured data.

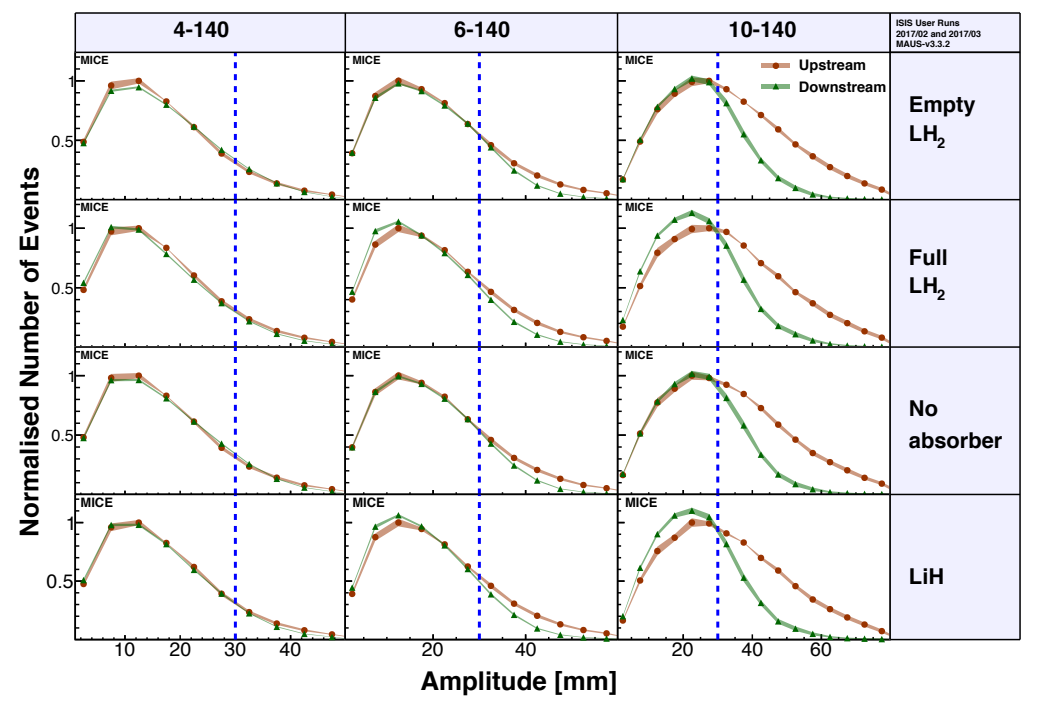

Figure 2: Muon amplitudes measured by MICE.

\section{Prospects}

Further settings are being explored in other MICE analysis having different momenta and different focussing properties. In particular, results including a lattice operating in solenoid mode 


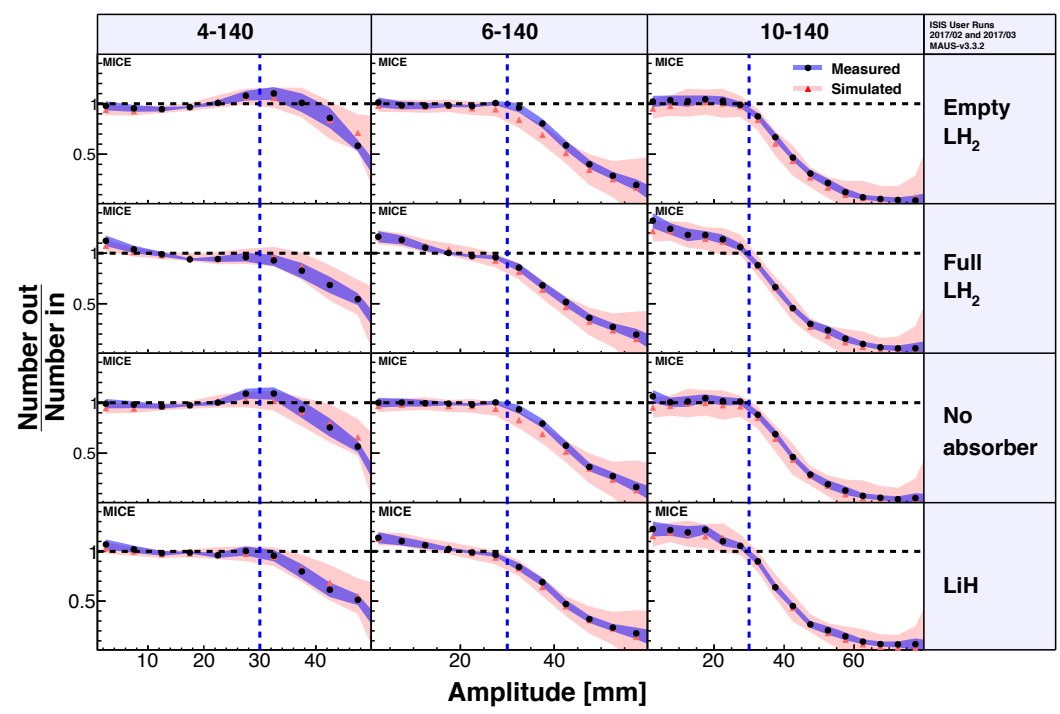

Figure 3: Downstream-to-upstream ratio of number of events in MICE.

and results showing RMS emittance are under study, presented in [4] and [5]. Studies are in progress for a follow-up experiment. Designs are under study for lattices with enhanced cooling at lower emittances, including RF. In particular, by including a dipole in the lattice arrangement, a modest position-energy correlation may be introduced into the beam (dispersion). By passing the beam through an appropriately aligned wedge-shaped absorber, higher energy particles will experience greater energy loss leading to exchange of emittance from longitudinal to transverse. Together with the transverse cooling described here, so-called '6D' cooling may be achieved. Studies of a muon source are underway. Using the proposed nuSTORM beam as a muon source is one particularly interesting proposal [6].

\section{Conclusions}

The Muon Ionisation Cooling Experiment has demonstrated conclusively the reduction of normalised emittance by ionisation cooling. Analysis of data for a number of different beam conditions is ongoing. Design work to pursue a follow-up experiment is ongoing.

\section{References}

[1] MICE collaboration, M. Bogomilov et al., Performance of the MICE diagnostic system, JINST $16(2021)$

[2] R. Asfandiyarov et al., MAUS: the MICE analysis user software, JINST 14 (2019)

[3] MICE collaboration, M. Bogomilov et al., Demonstration of cooling by the Muon Ionization Cooling Experiment, Nature 578, 53-59 (2020).

[4] P. Kyberd, Transverse Emittance Change and Canonical Angular Momentum Growth in MICE 'Solenoid Mode' with Muon Ionization Cooling, EPS-HEP2021 (2021). 
[5] P. Jurj, Normalized Transverse Emittance Reduction via Ionization Cooling in MICE 'Flip Mode', EPS-HEP2021 (2021).

[6] C. Rogers, NuSTORM accelerator: challenges and opportunities, IPAC2021 (2021). 